\title{
El proceso de reflexión orientado como una estrategia de investigación y formación: estudio longitudinal de caso
}

\author{
Carmen Peme-Aranega ${ }^{1}$ \\ Vicente Mellado ${ }^{2}$ \\ Ana Lía De Longhi ${ }^{3}$ \\ María Rosa Argañaraz ${ }^{4}$ \\ Constantino Ruiz ${ }^{5}$
}

Artículo recibido el 28 de noviembre de 2007 y aprobado el 27 de noviembre de 2008.

\section{The reflection guided process} as an investigations and formations' strategy: a longitudinal case study

Resumen: Describimos el caso de Mónica, una profesora de ciencias naturales de secundaria de Córdoba, Argentina, participante en un programa de desarrollo profesional basado en el proceso de reflexión orientado (colaborativo o tutorial). Analizamos el estado inicial y la evolución, durante cinco años, de sus concepciones didácticas y epistemológicas explícitas, de sus teorías en uso y de las implícitas subyacentes, y de sus modelos didácticos declarados (o deseables) y reales (o prácticos) en el aula. Los resultados indicaron que sus concepciones explícitas y sus modelos didácticos declarados iniciales eran mucho más avanzados que sus creencias implícitas y sus modelos prácticos. Al final de los cinco años, hubo bastante concordancia entre sus creencias explícitas e implícitas y sus modelos didácticos declarados y prácticos, ambos próximos a modelos constructivistas. Durante la investigación evolucionó gradualmente, con conflictos entre posiciones rivales, la manera en que, por medio del discurso áulico, orientaba la construcción del conocimiento de sus alumnos, organizaba y gestionaba la clase y evaluaba.

Palabras clave: Evaluación docente, desarrollo profesional, lineamientos.
Abstract: We describe the case of Mónica, a secondary education science teacher in Córdoba, Argentina, and participant in a program of professional development based on guided (collaborative or tutorial) reflection process. We analyze the initial state and evolution during five years of her explicit epistemological and educational conceptions, of her theories in use and the underlying implicit theories, and of her declared (desirable) and real (practical) educational models in the classroom. The results indicated that her explicit conceptions and her declared initial educational models were far in advanced of her implicit beliefs and her practice educational models. By the end of the five years, there was considerable concordance between her explicit and implicit beliefs, and between her declared and practical educational models, both close to constructivist models. During the period of the study, she gradually evolved, involving the resolution of conflicts between rival positions, about the way she oriented, through the classroom discourse, the students process of construction of knowledge, she organized and managed of the class and she evaluated.

Key words: Professional development, secondary education science teacher, collaborative guided reflection.

1 Departamento de Enseñanza de las Ciencias y la Tecnología, Facultad de Ciencias Exactas, Físicas y Naturales, Universidad Nacional de Córdoba, Argentina.

2 Departamento Didáctica Ciencias Experimentales y Matemáticas. Facultad de Educación, Universidad de Extremadura.

3 Departamento de Enseñanza de las Ciencias y la Tecnología; Facultad de Ciencias Exactas, Físicas y Naturales. Universidad Nacional de Córdoba, Argentina. Correo electrónico: analiadelonghi@yahoo.com.ar

4 Colegio Nacional de Montserrat, Universidad Nacional de Córdoba, Argentina.

5 Departamento Didáctica Ciencias Experimentales y Matemáticas, Facultad de Educación, Universidad de Extremadura. 


\section{Introducción}

El trabajo integra una línea de investigación (iniciada en 1993) con tres propósitos: desarrollar metodologías y procedimientos para estudiar algunas creencias (epistemológicas y didácticas, explícitas e implícitas) de docentes de ciencias (en formación y ejercicio), elaborar una "teoría sustantiva" para definir esas creencias y mejorar la relación teoría-práctica de docentes de ciencias, a partir de la sistematización de un Proceso de Reflexión Orientado, PRo, colaborativo o tutorial.

En el presente artículo estudiamos el caso de una profesora experimentada a cargo de la asignatura Ciencias Naturales en primer año del Ciclo Básico Unificado, CBU, en la provincia de Córdoba, República Argentina, a la que llamaremos Mónica. La profesora estudiada es docente de Física, se graduó en 1974 en una institución de formación docente no universitaria como Profesora de Matemáticas, Física y Astronomía. Su formación y los primeros años de trabajo estuvieron marcados por periodos de gobiernos militares. En ellos, la bibliografía de formación docente utilizada (especialmente fuera del ámbito universitario) estaba ideológicamente sesgada. Por otra parte, tanto el clima institucional como el áulico eran autoritarios y las estrategias didácticas empleadas no incluían trabajos participativos, como los grupales. En todo ese tiempo recibió un único modelo docente con el cual identificarse y debió adaptarse a normas y reglas rígidas de las que no podía salir. Todo ello fue estructurando su papel, sus formas de trabajo y sus esquemas de acción en el aula. Posteriormente, cursó una Postitulación en Ciencias Naturales en la Universidad Nacional de Córdoba, unc.
$\mathrm{Su}$ experiencia docente supera los veinticinco años, habiendo impartido asignaturas de Matemáticas y de Física en escuelas secundarias. En la institución en que se desarrolló el trabajo se desempeña desde el inicio de su carrera docente. Se trata de una escuela tradicional de gran prestigio, dependiente de la unc. Con motivo de la Reforma Educativa, que comenzó a implementarse en Argentina a partir de 1993, la institución fue adaptando su diseño curricular para introducir contenidos de física desde el primer año y asumió la asignatura Ciencias Naturales (que incluye esos contenidos) en tales cursos. La orientación curricular de la escuela es humanista. Sus alumnos, de edades entre once y doce años, pertenecen a un nivel social medio y medio alto.

Tratamos de analizar la incidencia mediadora de las creencias de la profesora en el complejo y sistémico proceso de enseñanza-aprendizaje en la etapa interactiva en el aula (Jackson, 1991) y la forma en que el PRo constituye una estrategia de desarrollo profesional que ayuda a solucionar algunos obstáculos y contribuye a la mejora de la práctica (Peme-Aranega, 2006a y 2006b y Vázquez, Jiménez y Mellado, 2007).

Durante un periodo de cinco años, analizamos el estado inicial y la evolución de sus concepciones didácticas y epistemológicas explícitas, así como de sus teorías en uso, sus creencias implícitas y sus modelos didácticos (tanto los declarados o deseables como los reales o puestos de manifiesto en el aula). Estudiamos la interacción entre las concepciones y la práctica discursiva áulica durante las distintas fases de la investigación. 
Muchas investigaciones señalan que los profesores de ciencias con experiencia tienen creencias y conocimientos prácticos personales muy estables (formados y consolidados en el transcurso de su actividad profesional) y que no cambian fácilmente ni sus concepciones ni sus prácticas docentes (Friedrichsen y Dana, 2005, Jeanpierre, Oberhauser y Freeman, 2005 y De Pro, 2006). Algunos señalan, como razones, el hecho de que se muestran satisfechos con modelos didácticos consolidados por su propia experiencia profesional, habiendo coherencia entre sus metas, sus concepciones y su conducta docente (McRobbie y Tobin, 1995). Otros marcan la existencia de condicionantes que refuerzan los modelos tradicionales y suponen obstáculos para cambiarlos (Jiménez y Wamba, 2003; Mellado, 2003; Reis y Galvao, 2004; Shwartz, Ben-Zvi y Hofstein, 2005; Peme-Aranega, 2006a y 2006b; Tobin, 1998; Tobin y McRobbie, 1996; Vázquez et al., 2006 y Verjovsky y Waldegg, 2005).

La mayoría de los profesores con experiencia considera que el cambio sólo merece la pena si les ayuda a resolver las situaciones prácticas cotidianas que se le plantean con sus alumnos. Para estos profesores, por tanto, la formación debe diseñarse como un proceso interno de "crecimiento" y de "desarrollo gradual" a partir de lo que ya piensan y hacen (Day, 1999), de los problemas reales de enseñanza y aprendizaje de las ciencias, de sus preocupaciones cotidianas y del contexto en el que desarrollan su actividad. Este trabajo se basa, justamente, en un PRo que tiene en cuenta las creencias de una docente, su práctica concreta, manifestada en las interacciones dis- cursivas en el aula, y sus necesidades de mejoramiento profesional gradual.

Estudios anteriores señalan que el desarrollo profesional se estimula por procesos sucesivos de autorregulación metacognitiva del docente basados en la reflexión, en la comprensión y en el control de lo que piensa, siente y hace (Copello y Sanmartí, 2001; Mellado et al., 2006 y Sanmartí, 2001).

El PRO empleado en este estudio genera procesos de metacognición importantes en la toma de conciencia de los problemas de enseñanza y aprendizaje que pueden ser mejorables, en la elaboración de nuevas actividades, materiales y propuestas de enseñanza (Powell y Anderson, 2002 y Sassi, Monroy y Testa, 2005) y en la puesta en práctica de las mismas en el propio contexto escolar.

El profesor, por tanto, no es un consumidor de conocimientos externos, sino un coproductor y un agente de cambio sobre los problemas que realmente le preocupan en sus clases (Cachapuz, 1995; Gil, Furió y Gavidia, 1998; Kimble, Yager y Yager, 2006).

Cada profesor evoluciona, partiendo de su propia situación y realidad hacia una mayor complejidad en la reflexión y en la práctica de la enseñanza de las ciencias (Vázquez, Jiménez y Mellado, 2007). Esto requiere tiempo y apoyos sostenidos para que el profesor pueda percibir la formación como una experiencia educativa válida para sí mismo y para sus clases (Peers, Diezmann y Watters, 2003; Sánchez y Valcárcel, 2000 y Tal et al., 2001).

Por ello, optamos en nuestro trabajo por un estudio que se extendió durante cinco años. Se trató de una investigación longitudinal, ya que cumplimentamos 
con los requerimientos que, según White y Arzi (2005), tiene un estudio educativo longitudinal: que transcurra al menos un año entre las distintas fases de recogida de datos y que la metodología permita una comparación de los resultados entre distintos periodos de tiempo. Coincidimos con numerosos autores (Baird et al., 1991; Lyons et al., 1997 y Roth, 1998) que (desde la investigación- acción) señalan que los estudios longitudinales de sus propios casos es, a mediano y largo plazo, una extraordinaria estrategia de desarrollo profesional.

La orientación constructivista considera que los profesores de Ciencias tienen concepciones sobre ellas y la forma de aprenderlas y enseñarlas profundamente arraigadas (Hewson y Hewson, 1989). El análisis y la reflexión tanto de las concepciones explícitas como de las implícitas en que se apoya la práctica en el aula es un primer paso para generar en los propios profesores unas concepciones y unas prácticas más adecuadas (Gil 1993; Hewson, 1993; Mellado, 1996; Peme-Aranega et al., 2005a y Peme-Aranega, 2006a y 2006b).

En los profesores con experiencia suele haber más coherencia que en los principiantes entre sus creencias tanto sobre la naturaleza de las ciencias (Ballenilla, 1992; Brickhouse, 1990; Gallagher, 1991; Lederman, 1999 y Tsai, 2002) como sobre su enseñanza-aprendizaje (Boulton-Lewis et al., 2001; Cronin-Jones, 1991; Dillon et al., 1994; Lorsbach et al., 1992; Mitchener y Anderson, 1989; Tobin y Espinet, 1989 y Wallace y Kan, 2004). Sin embargo, según el profesor y el contexto, puede haber discrepancias e, incluso, contradicciones entre sus concepciones y sus conductas docentes en el salón de clases al enseñar ciencias. Esto afectaría tanto a la creencias asociadas con la naturaleza de las ciencias (Duschl y Wright, 1989; Kang y Wallace, 2005 y Lederman y Zeidler, 1987) como a las relativas a su enseñanzaaprendizaje (Bol y Strage, 1996; Freire y Chorão, 1992; Huibregtse et al., 1994 y Lee y Porter, 1993) y a ambos tipos de creencias, epistemológicas y didácticas (Peme-Aranega, 2003 y Peme-Aranega et al., 2005b y 2007).

Los programas de desarrollo profesional deben tener en cuenta que los docentes con experiencia no suelen hacer cambios radicales, sino que van sucesivamente incorporando y poniendo en práctica aquello que consideran positivo para el aprendizaje de sus alumnos. Estos cambios suelen ser lentos y graduales (Tal et al., 2001), con frecuentes desfases (Da Silva et al., 2007; Furió y Carnicer, 2002; Marx et al., 1998 y Pacca, Pacca y Villani, 1996) y raramente implican el completo abandono de sus modelos didácticos en favor de otros nuevos, sino que más bien son adquisiciones y retenciones parciales (Gunstone y Nothfield, 1994; López Ruiz, 2000; Luna y García, 2003 y Valcárcel y Sánchez, 2000).

\section{Objetivos}

Esta investigación etnográfica longitudinal aborda dos problemas: ¿Es posible hacer evolucionar los modelos didácticos de docentes de ciencias desde un proceso formativo de reflexión orientado, tutorial o colaborativo? ¿Es posible sistematizar este proceso basándolo en el conocimiento de sus creencias epistemológicas y didácticas? 
En este artículo se presenta parte de esta línea de trabajo, cuyas finalidades hacen a la investigación y a la formación docente continua. Los objetivos que se abordan se relacionan con conocer, analizar e interpretar el estado inicial y la evolución de las creencias (epistemológicas y didácticas, explícitas e implícitas) de una profesora de ciencias de Ciclo Básico Unificado, sus teorías en uso, las implícitas subyacentes, sus modelos didácticos (deseables y reales) y los obstáculos para su desarrollo profesional. Además, formular hipótesis acerca de sus niveles profesionales de partida y su progresión; implementar un programa de formación que, tomando como eje el PRo, desarrolle otras actividades de formación complementarias y consensuadas y analizar e interpretar la evolución y factores que inciden en su desarrollo profesional.

\section{Metodología}

La investigación comenzó con una fase exploratoria inicial (2001), durante la cual se hizo un registro de lo sucedido en cuatro clases y se inició la recopilación de datos relacionados con el conocimiento de las creencias explícitas de la profesora. Luego de esta fase exploratoria inicial, se empleó un proceso de investigación cíclico de cinco fases (F) cada uno. Al finalizar la última $\mathrm{F}$ se reiniciaba el ciclo.

Las actividades fueron:

- conocimiento de creencias epistemológicas y didácticas explícitas (F1).

- conocimiento de teorías en uso (F2).

- conocimiento de las creencias implícitas subyacentes (F3).

- identificación de modelos didácticos personales deseables y reales (F4).
- evaluación del nivel profesional (de partida o su progresión), detección de obstáculos para su desarrollo y establecimiento de hipótesis de progresión (F5).

El PRO se realizó en todos los ciclos (uno a cinco) y fue el eje del proceso formativo y del diseño y la ejecución de otras actividades complementarias de formación acordes con las necesidades de Mónica y los obstáculos para su desarrollo profesional.

En la Figura 1 se presenta un esquema de las F que componían cada uno de los ciclos. Así:

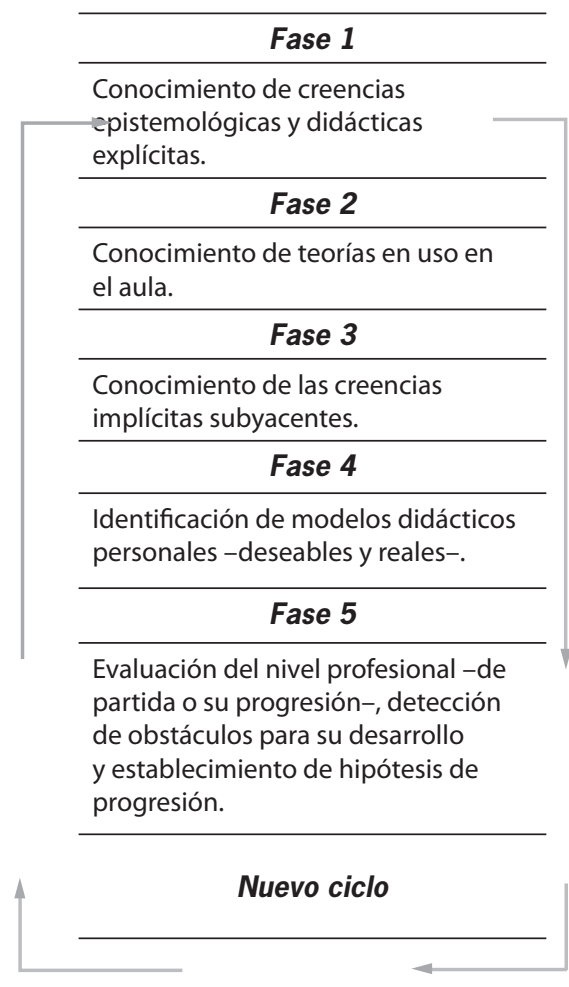

Figura 1: Fases que formaban parte de los ciclos.

Proceso de reflexión orientado. Diseñoy ejecución de actividades complementarias de formación. 
El estudio, que duró cinco años, incluyó cinco ciclos, realizados en los cursos lectivos de Argentina correspondientes a 2002, 2003, 2004 (dos ciclos) y 2005. Cada nuevo ciclo implicaba una reiteración de las F 2, 3, 4 y 5, del proceso de reflexión orientado y de la planificación de actividades.

El instrumento interpretativo de todos los datos fue la "teoría sustantiva", desarrollada por Peme-Aranega et al. (1999), integrada por dos categorías conceptuales teóricas (c): Didáctica de las ciencias y Epistemología (o Imagen de las ciencias). Cada categoría está, a su vez, conformada por subcategorías (sc) (Tabla 1).

Las distintas sc, a su vez, están compuestas por dimensiones de análisis. Las dimensiones son aspectos de dichas sc expresados en términos de polos opuestos; en el polo positivo se encuentran las concepciones didácticas y episte- mológicas más próximas a las actuales posiciones de la comunidad científica que trabaja en el área (Peme-Aranega, 2006a y 2006b).

Las creencias explícitas se infirieron de:

- Entrevistas semiestructuradas, realizadas al comienzo del estudio; éstas se sometieron a un "análisis de contenido" (Bardin, 1986) y, posteriormente, fueron interpretadas con la "teoría sustantiva", por un acuerdo entre dos jueces.

- El Inventario de Creencias Pedagógicas y Científicas, Inpecip, elaborado por Porlán (1989).

- el Inventario de Creencias Epistemológicas y Didácticas, ICDE (Peme-Aranega et al.,1999), que fue estudiado psicométricamente y utilizado en diferentes estudios con profesores de ciencias en pequeñas y grandes muestras (Mellado et al., 2003; Peme-Aranega, 2001; PemeAranega y Baquero, 2001a y 2001b).

Sistema de subcategorías (sc) correspondiente a la categoría Epistemología (o Imagen de las ciencias):

sci: Caracterización de las ciencias, de los científicos, del conocimiento y de las teorías científicas. scii: Caracterización de progreso de las ciencias. Importancia de factores históricos, sociales, políticos en el desarrollo de las ciencias.

sciii: Metodologías. Papel de la observación, de la experimentación, de los datos, de las teorías. sciv: Concepción de teorías y de teorías rivales. scv: Realismo, instrumentalismo, interaccionismo.

Sistema de subcategorías (sc) correspondiente a la categoría Didáctica de las ciencias:

scl: Relación conocimiento cotidiano y construcción del conocimiento científico.

scll: Caracterización y relaciones entre conocimiento cotidiano, conocimiento escolar y conocimiento científico.

scill: Caracterización del conocimiento y del aprendizaje.

sciv: Caracterización del aprendizaje científico escolar.

scv: Caracterización de la enseñanza, de la didáctica, de la profesión y de la formación del profesorado de ciencias experimentales.

scvl: Conceptualización de la enseñanza de las ciencias.

scvil: Conceptualización de la función docente.

scVIII: Concepción de la enseñanza en general e institucional en particular.

scix: Concepción de la programación.

scx: Concepción de la metodología.

scxl: Concepción de la evaluación.

Tabla 1: Subcategorías correspondientes a las categorías Epistemología y didáctica de la teoría sustantiva. 
Los dos Inventarios se aplicaron en tres oportunidades: al inicio de la investigación (2001), durante la misma (2003) y en el último año del trabajo (2005).

Las teorías en uso se dedujeron del discurso interactivo en el aula, el cual, como en otros estudios (De Longhi, 2000; Peme-Aranega, 2006a y 2006b y Peme-Aranega et al., 2005 y 2007), fue una importante fuente de datos. Se analizaron diecinueve clases a las que se asistía como observador participante.

Se construyeron unidades de análisis de las clases. La investigadora y un evaluador externo acordaron la partición de cada clase, lo que fue consensuado con la docente que la había desarrollado. Con esas unidades se elaboraron los primeros protocolos de análisis. Posteriormente, se consideraron los comportamientos verbales incluidos en cada unidad (utilizando una lista de comportamientos verbales de clases que se venía elaborando desde estudios anteriores, Peme-Aranega, 2006a). Se confeccionaron con ellos los segundos Protocolos de análisis (consensuados con un evaluador externo y la docente).

En cada clase se consideraron los comportamientos que integraban las distintas unidades y las conductas secuenciadas presentes en cada unidad. Del cuadro de frecuencias de comportamientos y de secuencias más repetidas se derivaron las teorías en uso de las profesoras en cada uno de los ciclos.

Los comportamientos se interpretaron ubicándolos en la c, en las sc y en las dimensiones de análisis de la "teoría sustantiva” (presentada en la Tabla 1). El acuerdo investigadora-evaluador externo-docente condujo a la confección de los terceros protocolos de análisis (unidades, conductas y significado), deduciéndose así las teorías implícitas manifestadas en cada ciclo. De las creencias explícitas se infirieron los modelos didácticos personales declarados o deseables (lo que las docentes "decían") al inicio del trabajo, en 2003 y en 2005. Finalizado el proceso de los terceros protocolos de análisis, se identificaron los modelos didácticos reales (lo que la profesora "hacía"), correspondientes a las teorías en uso en los distintos ciclos. Las creencias implícitas daban cuenta de las teorías en que se apoyaban esos modelos didácticos manifestados en el aula.

El marco de referencia para identificar los modelos fue un trabajo teórico basado en estudios de diversos autores (Peme-Aranega, 2006a). La comparación de las creencias explícitas e implícitas iniciales permitió inferir el nivel profesional de partida y determinar obstáculos para el desarrollo profesional de la docente. El desarrollo profesional se obtuvo comparando el nivel profesional de partida con las posteriores diferencias entre las creencias explícitas e implícitas.

Como ya se dijo, el pro fue el eje de la formación de Mónica en el estudio. Abarcó todo el proceso de la investigación y se basó en los consensos investigadora principal-docente acerca de la "teoría sustantiva", los distintos protocolos de análisis, las creencias explícitas, las teorías en uso e implícitas, los modelos (deseables y reales), el nivel profesional de partida, las hipótesis de progresión, los obstáculos y las actividades formativas complementarias, los resultados encontrados, la evolución manifestada 
en el transcurso de los ciclos; las conclusiones y las implicaciones.

También se consensuaron y llevaron a cabo actividades de formación, complementarias al PRo, adaptadas a las necesidades, obstáculos y problemas prácticos de Mónica. Éstas fueron:

- Lecturas y discusiones de distintos modelos o enfoques didácticos utilizados en la literatura de didáctica de las ciencias (Porlán y Rivero, 1998 y Pozo y Gómez Crespo, 1998). Esta actividad le permitió identificar sus propios modelos didácticos (deseables y reales).

- Un taller formativo sobre la planificación de unidades didácticas en temas concretos del currículo.

- La planificación conjunta (durante el cuarto ciclo) de las dos últimas clases del quinto ciclo, empleando la metodología de investigación dirigida.

Estas dos últimas actividades se realizaron, consensuadamente, debido a que la planificación de unidades didácticas es un importante aspecto del desarrollo profesional (De Pro, 1999) además de indicar que el profesor comienza a cambiar hacia orientaciones más actualizadas (Bartholomew y Osborne, 2004 y Martínez-Losada y García-Barros, 2005).

\section{Resultados \\ El inicio de la investigación}

En la entrevista semiestructurada y en las respuestas al ICDE y al Inpecip, Mónica evidenció creencias didácticas y epistemológicas explícitas iniciales bastante actualizadas.

En los aspectos psicológicos (incluidos en la categoría Didáctica de las ciencias) manifestó un constructivismo simplificado (López Ruiz, 1994). En lo que respecta a los aspectos curriculares y organizativos (de la misma categoría), la visión de la profesora también fue constructivista. No obstante, dejó en evidencia limitaciones y posiciones menos actualizadas con respecto a la concepción de didáctica, a la función docente, a la programación, a la evaluación y a la metodología. También mostró ciertas tendencias a un empirismo metodológico y a considerar que el conocimiento escolar es un simple reemplazo del cotidiano.

En lo que hace a la categoría Imagen de las ciencias mostró una perspectiva relativista; una concepción metodológica próxima a los planteamientos falsacionistas; visiones contextualizadas de las ciencias y de su progreso y una visión actualizada acerca de las teorías, (aceptando la coexistencia de marcos teóricos rivales). Sus limitaciones se asociaron con un cierto relativismo empírico y con sus indefiniciones respecto al papel de las observaciones y de las hipótesis en la metodología de la investigación. Sus enfoques no lograron concretarse en un realismo crítico.

El modelo didáctico inicial deseable (o declarado) de Mónica era de carácter mixto: mostraba una superación del enfoque tradicional, una entrada al modelo tecnológico y la presencia de escasos rasgos de enfoques constructivistas.

En lo que se refiere a sus interacciones discursivas en el aula, sus teorías en uso al comienzo del estudio dejaron en evidencia la existencia de modelos prácticos (o reales) menos evolucionados que los declarados: un traspaso desde el enfoque tradicional (del que estaba saliendo) al modelo tecnológico (al que iniciaba su entrada).

En lo que respecta a los aspectos epistemológicos, sus modelos reales 
daban cuenta de concepciones implícitas absolutistas del conocimiento y una orientación única, rígida e infalible de la metodología científica.

Desde el punto de vista didáctico, los comportamientos verbales de Mónica durante el proceso interactivo se categorizaron en tres grupos:

- Los que hacían a la construcción del conocimiento por parte de los alumnos.

- Los relacionados con la organización y con la gestión de las actividades.

- Los vinculados con la evaluación.

Al comienzo del trabajo, la profesora mostró, respecto a la forma de orientar la construcción del conocimiento de sus alumnos en el aula, teorías en uso centradas en sí misma (no en ellos) y basadas en la exposición de conocimientos elaborados. Tales exposiciones, casi siempre exclusivas para toda la clase, eran desarrolladas siguiendo la lógica de sus propios razonamientos mediante un discurso continuado y sin tener en cuenta el grado de significación para los estudiantes. Trataba de mantener el hilo de ese discurso, sólo interrumpido por preguntas hechas a los estudiantes en búsqueda de respuestas que le permitieran volver a su idea conductora o por cuestiones que ella misma se contestaba para poder retomarla; reforzando positivamente a los alumnos cuando lo hacían según lo esperado y corrigiéndolos cuando esto no ocurría, como forma de encauzarlos. En principio, Mónica evidenció también un cierto absolutismo epistemológico y didáctico, lo que supuso un obstáculo para su desarrollo profesional (Porlán, Rivero y Martín, 1997 y Jiménez y Wamba, 2003).

De acuerdo con la gestión de las actividades de los estudiantes en el aula, Mónica dejó en evidencia, al comenzar la investigación, la existencia de dos teorías en uso rivales simultáneas: por un lado, las que se caracterizaban por una conducción netamente heteroestructurante, basada en la directividad y en la que el grupo de alumnos era tratado en forma homogénea; en sentido contrario manifestaba otras, de orientación interestructurante, basadas en la orientación y en el manejo diversificado del grupo (Not, 1983). Los indicadores verbales de sus creencias implícitas acerca de la evaluación fueron prácticamente nulos, sólo utilizó preguntas para considerar las respuestas de los alumnos.

En resumen, al inicio del trabajo con la profesora, existía una gran diferencia entre sus concepciones declaradas y las implícitas; entre lo que quería hacer y lo que realmente hacía en el aula. Como se dijo, sus modelos reales estaban retrasados respecto a los declarados como deseables.

Se puede concluir que al inicio Mónica estaba insatisfecha con las formas tradicionales de enseñanza-aprendizaje $y$, aun cuando estaba iniciando un proceso de cambio, mostraba una concepción predominantemente centrada en el profesor.

\section{Evolución de Mónica durante los cinco años de la investigación}

Las creencias epistemológicas explícitas de Mónica se aproximaron cada vez más a los extremos positivos de las diferentes dimensiones incluidas en todas las subcategorías; sin embargo, no logró consolidar una perspectiva realista crítica.

De acuerdo con sus creencias didácticas explícitas, alcanzó -al finalizar el estudio- un constructivismo simplificado (López Ruiz, 1994). Pero no logró 
superar definitivamente su inductivismo empirista y mantuvo también limitaciones en sus creencias declaradas asociadas a una visión algo tecnocrática de la didáctica y conductista de la evaluación. Respecto a la programación, continuó también dando demasiada importancia a la organización jerárquica de los objetivos.

En la práctica en la sala de clase y, en términos generales, podemos decir que nuestra docente partió de un conflicto entre teorías rivales hasta el triunfo final de estrategias interestructurantes (Not, 1983): relativización del conocimiento y orientación en la resolución problemas. Las idas y venidas alargaron el proceso. Su cambio se asemejó a los progresos lakatianos (Mellado et al., 2006).

En que se refiere a los comportamientos discursivos relacionados con la construcción del conocimiento de sus estudiantes, Mónica dejó en evidencia, al inicio y como ya se dijo, una rivalidad entre estrategias netamente heteroestructurantes (Not, 1983), como la exposición y otras más interestructurantes, como los trabajos grupales.

Pero poco a poco las teorías heteroestructurantes fueron cediendo paso a las interestructurantes y la evolución se fue viendo con mayor claridad: de manera paulatina, Mónica fue reemplazando su exposición continuada y unidireccional por el empleo del diálogo y otras formas menos directivas, enriqueciendo su significado. Fue limitando sus intervenciones y buscando una mayor participación de los estudiantes y de los grupos tratando que éstos, junto con ella, elaboraran el conocimiento escolar y recordaran conceptos previamente desarrollados. Puso en juego los procesos cognitivos de los alumnos (tanto de reflexionar como de relacionar y sintetizar, segundo ciclo). Además de aceptar los aportes estudiantiles y precisarlos (segundo ciclo), mostró también la valoración que les daba, empleando un recurso integrador grupal como el diagrama conceptual (cuarto ciclo). Fue también reconociendo las contribuciones de los estudiantes y admitiendo que emplearan distintas formas de resolver situaciones (tercer ciclo). Por medio de preguntas o permitiéndoles explicar fue indagando sus conceptos previos y sometiendo al juicio del grupo los aportes individuales (cuarto ciclo). Además, extendió el diálogo al grupo, aceptando todas las respuestas y poniéndolas a consideración. Más adelante (quinto ciclo) solicitó a los alumnos mayor precisión en sus respuestas; extendió la aceptación de sus contribuciones a nuevos comportamientos verbales (como pedirles que formularan hipótesis o resolvieran sus propias propuestas) y limitó sus exposiciones a dar sólo las informaciones necesarias, adaptándolas a situaciones diferenciadas. En este ciclo, también surgieron los dibujos como recursos tendientes a soportar su exposición o la explicación dada por los alumnos.

En las dos últimas clases de ese quinto ciclo, preparadas en forma conjunta, se registró el mayor número de secuencias interactivas verbales nuevas asociadas al estudio de problemas experimentales y a las formas de incentivar los procesos incluidos en su resolución (formular hipótesis, encontrar respuestas a las propias propuestas, imaginar, precisar las mediciones experimentales y someter los procedimientos empleados al juicio del grupo, etc.) y unidades 
que mostraban un papel personal de acompañamiento de los estudiantes (oficiar de facilitadora de situaciones conjeturables, ayudarles a resolver problemas técnicos en el laboratorio, acompañar las participaciones verbales con dibujos y gráficos). Así pudo verse cómo el estudio finalizaba con las primeras implementaciones que hizo Mónica del estudio dirigido de problemas.

En lo que hace a la gestión de las actividades áulicas, Mónica mostró desde el comienzo y hasta el cuarto ciclo teorías contrapuestas; algunas directivas y correctoras, como manifestar que quien determinaba, dirigía y fijaba los tiempos de las actividades era ella y controlar la disciplina, las que, devenidas del inicio del estudio, se continuaron durante el segundo ciclo. Luego (tercer ciclo), se observaron con mayor frecuencia conductas verbales que eran signos de la búsqueda de procesos más autónomos en los alumnos, como exponer un tema que ellos mismos habían preparado, solicitarles criterios para evaluar la actividad y hacerles valorar a sus grupos de compañeros: heteroevaluación. En el cuarto ciclo, aún existiendo comportamientos de patrones directivos, fueron más frecuentes los contrarios. También aparecieron otros de flexibilización de los tiempos planificados en función de los alumnos, dándoles oportunidad de elegir con qué actividad seguir o cómo hacerlo. En el quinto ciclo casi no se manifestaron las pautas directivas y sí aquellas incentivadoras de una mayor autonomía y consideración de las necesidades, los intereses, los tiempos y las decisiones de los estudiantes. Apareció también otro comportamiento verbal relacionado con la búsqueda de autonomía: inducir a los estudiantes a encontrar respuestas a sus propias propuestas. La conducta verbal asociada al tratar de que los alumnos comprendieran el significado de una actividad nueva estuvo, al inicio de la investigación, ligada a las teorías verbales directivas; pero, a medida que el trabajo continuó, se relacionó con otras más orientadoras y menos modeladoras.

Referente a la evaluación, recién en el tercer ciclo del estudio aparecieron unidades y secuencias conductuales discursivas interactivas asociadas con dicho proceso, como, por ejemplo, el uso de la heteroevaluación y solicitarle a los alumnos criterios para evaluar una actividad, aun cuando finalmente reforzó los que ella misma consideró adecuados. Estos poco indicadores no permiten analizar la evolución de sus teorías en uso acerca de este aspecto.

El análisis cuantitativo de las teorías implícitas subyacentes (limitadas sólo a la construcción del conocimiento en el aula) surgió de la comparación de las concepciones actualizadas -hoy consideradas como tal por la comunidad que trabaja en didáctica de las ciencias (+) y las desactualizadas según la misma comunidad (-). La interpretación se hizo, como se ha dicho, empleando la "teoría sustantiva” (Peme-Aranega et al., 1999). Se contabilizaron las conductas $+y$ - que, en cada clase de la profesora, superaban el 5\%. Se calculó luego el porcentaje de comportamientos que evidenciaban concepciones implícitas actualizadas (+) y desactualizadas (-) en cada uno de los cinco ciclos. En la figura 2 se representa la evolución de las teorías implícitas de Mónica respecto a la construcción del conocimiento en el aula en el transcurso de los cinco ciclos. 
En cada uno de los ciclos se identifican dos direcciones de comportamientos discursivos: a) se consideran positivos (+) aquellos que van desde el docente (D) al alumno (A) y b) negativos (-) los que van de modo directo desde el profesor (D) al objeto de conocimiento (O). Una mayoría de las primeras refleja un modelo centrado en los estudiantes; en cambio, un mayor número de las segundas manifiesta un enfoque centrado en la transmisión del contenido por parte de la profesora.

De este análisis se concluyó que sus teorías implícitas respecto a la construcción del conocimiento en el aula evolucionaron (aumentaron los comportamientos discursivos positivos $y$ disminuyeron los negativos). En los tres primeros ciclos hubo poca diferencia entre las teorías implícitas heteroestructurantes sobre la construcción del conocimiento -que iban de la docente (D) al objeto de conocimiento (OC) hasta llegar a los estudiantes- y las interestructurantes -que circulaban desde la docente (D) hacia los alumnos (A). La diferencia entre ambos tipos de direcciones y a favor de la segunda se observó con claridad en los dos últimos ciclos.

En Mónica, profesora con experiencia, el PRO tuvo que incidir sobre prácticas demasiado estructuradas en el transcurso del tiempo en lo que respecta a la forma de orientar la construcción de conocimientos por parte de los alumnos. El pasaje de una orientación centrada en sí misma y en la materia a otra que tomase como eje a los alumnos y a su aprendizaje no fue un proceso fácil.

En concordancia con todo lo anterior, concluimos que los modelos didácticos reales de Mónica manifestados concretamente en el aula evolucionaron, en todos los aspectos, desde un modelo real inicial (que se encontraba pasando del tradicional al tecnológico) hacia un modelo final que iba en la dirección del investigativo, pero que mantuvo características del tecnológico al que se sumaron rasgos de otros modelos constructivistas: tanto del expositivo en el sentido ausubeliano como del de conflicto cognitivo.

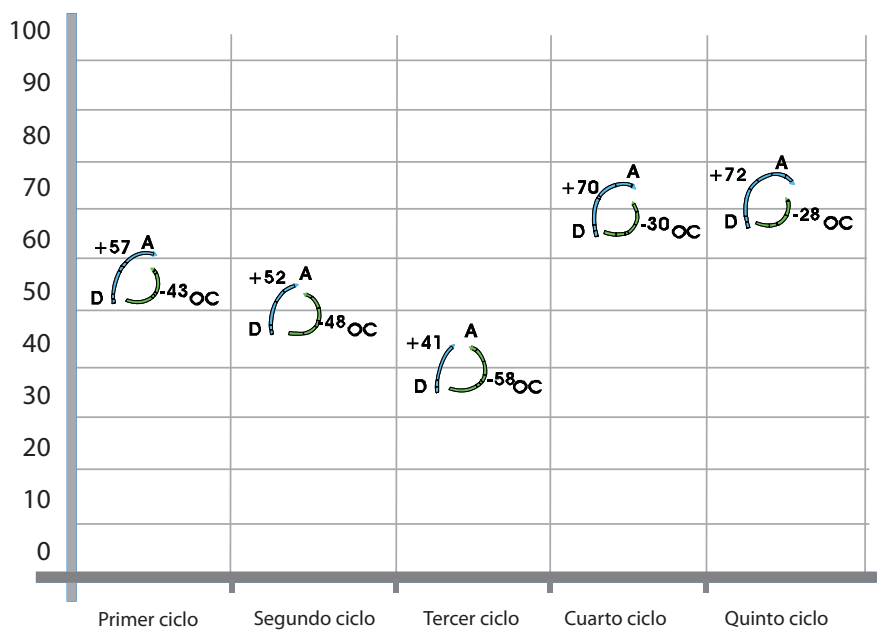

Figura 2: Evolución de las teorías implícitas de Mónica sobre la construcción del conocimiento en los cinco ciclos de la investigación. 


\section{Conclusiones}

- Los procedimientos empleados en el estudio longitudinal resultaron adecuados para conocer las concepciones epistemológicas y didácticas explícitas e implícitas de Mónica, sus teorías en uso y las implícitas subyacentes (inferidas del discurso interactivo en el aula), sus modelos didácticos (deseables y reales). Éstos también permitieron determinar sus niveles profesionales de partida y su evolución en los cinco ciclos de la investigación y conocer algunos obstáculos para su desarrollo. La "teoría sustantiva" permitió interpretar datos de distintas fuentes, en diferentes contextos y momentos en relación con las creencias de la nuestra docente-caso.

- El proceso de reflexión orientado (basado en sus creencias y tomando como criterio de sistematización los consensos interpretativos adaptados a la docente) constituyó una metodología de formación que permitió desarrollar actividades complementarias, hacer evolucionar tanto los modelos deseables o declarados de Mónica como los reales, manifestados en su actividad en el aula, contribuyendo al mejoramiento de sus prácticas discursivas y a su desarrollo profesional.

- Además de nuestras propias investigaciones, otros estudios anteriores (Bailey, Scantlebury y Johnson, 1999) permitieron concluir que la reflexión colaborativa (PRO) es un procedimiento esencial en el desarrollo profesional del profesorado.

- Mónica mostró diferencias iniciales entre sus concepciones didácticas y epistemológicas manifiestas y las creencias implícitas subyacentes, entre sus estructuras conceptuales y los esquemas de acción y entre los modelos deseables y reales. Su nivel profesional académico de partida era superior al profesional práctico.

- Algunas investigaciones señalan que los profesores con experiencia suelen tener conductas docentes más avanzadas y actualizadas que lo que expresan en sus concepciones explícitas (Freitas, Jiménez y Mellado, 2004; Lucas y Vasconcelos, 2005; Pavón, 1996 y Rodríguez y López y Mota, 2006). El caso de Mónica no coincide con los resultados anteriores, sino que tiene muchos rasgos comunes con los profesores de ciencias con años de experiencia analizados por Luna y García (2003) que, en el caso estudiado, encontraron divergencias entre lo que el profesor sabe que tiene que hacer y lo que realmente hace. También se asemeja a las conclusiones de Reyes, Salcedo y Perafán (2001), quienes observaron en la profesora analizada deseos de cambio que no se traducían necesariamente en acciones en el aula.

- Durante el estudio, Mónica fue delineando más su modelo deseable, que iba abandonando rasgos del tecnológico y adoptando características de los constructivistas. Los análisis y discusiones mantenidas durante el proceso reflexivo colaborativo le fueron dando elementos conceptuales desde los cuales fundamentar sus decisiones. El trabajo con los comportamientos verbales asociados a concepciones implícitas, en el cual las teorías en uso se confrontaban con raíces más profundas, le fueron permitiendo tomar conciencia de su logicismo didáctico y del verdadero valor que le daba a las concepciones espontáneas al momento de su manejo en el aula.

- Trabajar con Mónica significó trabajar desde los conflictos entre creencias implícitas opuestas para que fuera optando, desde la razón, por alternativas diferentes. Cada toma de conciencia aumentaba su angustia ante los cambios, esgrimiendo mecanismos defensivos para tales sentimientos. Los viejos esquemas le habían servido y eran reconocidos por su entorno. Los nuevos no habían sido probados y no sabía cómo iban a ser recibidos. A medida que reflexionaba sobre su práctica, iba buscando formas 
de cambiarla, probando y evaluando sus resultados. Las limitaciones de su medio empezaron a dejar de ser sentidas como tales y los conflictos entre creencias encontradas se fueron definiendo. Su camino de idas y venidas se fue encauzando, pero le faltaban elementos para sentirse segura de aplicar el modelo de investigación dirigida por el cual finalmente optó, a nivel explícito, y que se animó a planificar e implementar con orientación. El análisis y la reflexión sobre esas clases le brindaron elementos que ella consideraba necesarios para seguir avanzando. El modelo real, finalmente alcanzado, es una prueba de su desprendimiento de los modelos tradicional y tecnológico y una muestra de su estado actual de transición, en el cual coexisten rasgos de tres modelos constructivistas. La distancia entre su modelo deseable y el real se ha estrechado.

- Estas conclusiones coinciden con lo señalado por otros autores (Reyes, Salcedo y Perafán, 2001) cuando consideran que el trabajo colaborativo con profesores brinda apoyos importantes a sus procesos reflexivos y a las actividades de planificación docente, aumentando también su autoestima.

- A partir de lo anterior, consideramos que la cultura tradicional de las escuelas supone una dificultad para llevar a cabo una enseñanza más innovadora para muchos profesores de ciencias con años de experiencia docente. (Reyes, Salcedo y Perafán, 2001 y Verjovsky y Waldegg, 2005).

- Los principales obstáculos a trabajar en el PRo, detectados al comienzo de la investigación, eran el absolutismo epistemológico y didáctico de Mónica. En tanto avanzamos en el trabajo, fuimos percibiendo otros que los reforzaban, provenientes de su contexto pasado y presente, de su experiencia y de su personalidad. Para ella, su formación académica, que consideraba tradicional, constituía su mayor limitante y esto le provocaba inseguridad. Tuvimos que buscar formas de resolverla satisfaciendo sus necesidades académicas, ya que los conocimientos constituían la base desde la cual la profesora iniciaba los cambios. También debíamos trabajar la incidencia del contexto autoritario en que se había formado y los modelos docentes incorporados en ese periodo, los cuales eran conductistas, directivos y no reflexivos. Actuamos sobre ellos de modo indirecto generando, como contrapartida, un clima democrático de discusión y consensos que le permitiese reflexionar y ser consciente de las limitaciones. Éste fue un primer paso para su superación y le permitió sacar a la luz cada vez más elementos para evolucionar. A medida que fue cobrando seguridad, empezó a cuestionarse sobre aspectos del contexto escolar con los que estaba identificada para, finalmente, buscar formas de cambiarlos. En tanto fue reflexionando y probando alternativas, las restricciones del medio dejaron de ser vivenciadas como tales, los conflictos entre opuestos se fueron definiendo y orientando a nivel explícito hacia el modelo investigativo.

- La planificación y análisis conjuntos de clases, su implementación y la reflexión sobre ellas como estrategia para su formación docente complementaria al PRO le dieron pruebas y seguridad para continuar evolucionando.

- Así, su proceso de investigación sobre su práctica, de evolución lakatiana, tiene en la actualidad una nueva dirección.

- Desde el marco teórico de la didáctica de las ciencias experimentales, los resultados de esta investigación contribuyen a confirmar la importancia de trabajar sobre las creencias didácticas y epistemológicas de los docentes, ya que ellas contextualizan y determinan, en gran medida, lo que se planifica, desarrolla y evalúa en las clases de ciencias. 
El alcance de esta línea de trabajo se inserta en las didácticas especiales, ya que las características del objeto las determinan.

\section{Implicaciones}

Creemos que el PRO incide de manera muy diferente en los profesores participantes según su formación inicial, su experiencia docente, su contexto escolar, etc. Esto nos lleva a la necesidad de individualizar el desarrollo profesional, según las características personales y sociales del profesorado con el que se colabore y a destacar la importancia de programas específicos para la iniciación a la docencia, una etapa en la que se están formando y consolidando las estrategias y rutinas en el aula que, posteriormente, serán mucho más difíciles de cambiar.

Queremos resaltar que, en futuras investigaciones, sería necesario relacionar el desarrollo profesional del profesorado y el aprendizaje del alumnado, incidiendo en especial en el proceso de construcción del conocimiento por parte de los estudiantes (sus dificultades, su progresión, la construcción de significados, la evaluación, etc.), como también en el conocimiento didáctico del contenido por parte de los docentes (Reyes y Garritz, 2006 y Shulman, 1986). Pensamos, además, que sería importante centrarse en temas concretos de ciencias del currículo escolar y aprovechar las investigaciones ya realizadas sobre la construcción y progresión del conocimiento escolar en estos temas (Banet y Ayuso, 2000 y Jiménez-Aleixandre y Reigosa, 2006), para que los profesores participantes tengan referencias de las que partir en el proceso de construcción del conocimiento del alumnado.
Finalmente, queremos decir, y no por hacer un juego de palabras, que este estudio ha sido una investigación que empleó la formación docente y una formación docente que empleó la investigación. Ello implicó un trabajo en profundidad sobre aspectos que hacen a la profesionalización del profesor que buscaba estrechar la relación no sólo teoría-práctica, sino también el circuito de esa relación mediado por la reflexión.

\section{Agradecimiento}

Este trabajo ha sido financiado por los Proyectos de Investigación del Ministerio de Educación y Ciencia de España (SEJ2006-04175), de la Consejería de Educación de la Junta de Extremadura (PRI06A039) y de la Secretaría de Ciencia y Tecnología de la Universidad Nacional de Córdoba (05 - I422, años 2001 -2003).

\section{Referencias bibliográficas}

Bailey, B. L., Scantlebury, K. C. y Johnson, E. M. (1999). Encouraging the beginning of equitable science teaching practice: Collaboration is the key. Journal of Science Teacher Education, 10(3), 159-173.

Baird, J. R., Fensham, P. J., Gunstone, R. F. y White, R. T. (1991). The importance of reflection in improving science teaching and learning. Journal of Research in Science Teaching, 28(2), 163-182. Ballenilla, F. (1992). El cambio de modelo didáctico, un proceso complejo. Investigación en la Escuela, 18, 43-68.

Banet, E. y Ayuso, E. (2000). Teaching genetics at secondary school: a strategy for teaching about the location of inheritance information. Science Education, 84(3), 313-351. 
Bardin, L. (1986). Análisis de contenido. Madrid: Akal.

Bartholomew, H. y Osborne, J. (2004). Teaching students "ideas about science": five dimensions of effective practice. Science Education, 88(5), 655-682.

Bol, L. y Strage, A. (1996). The contradiction between teachers' instructional goals and their assessment practices in high school biology courses. Science Education, 80(2), 145-163.

Boulton-Lewis, G. M., Smith, D. J. H., Mccrindle, A. R., Burnet, P. C. y Campbell, K. J. (2001). Secondary teachers' conceptions of teaching and learning. Learning and Instruction, 11(1), 3551.

Brickhouse, N. W. (1990). Teachers' beliefs about the nature of science and their relationship to classroom practice. Journal of Teacher Education, 41(3), 53-62.

Cachapuz, A. (1995). Da investigação sobre e para professores à investigação com e pelos professores de ciências. En L. J. Blanco y V. Mellado (eds.), $L a$ formación del profesorado de ciencias y matemáticas en España y Portugal (pp. 243-254). Badajoz: Diputación Provincial.

Copello, M. I. y Sanmartí, N. (2001). Fundamentos de un modelo de formación permanente del profesorado de ciencias centrado en la reflexión dialógica sobre las concepciones y las prácticas. Enseñanza de las Ciencias, 19(2), 269-283.

Cronin-Jones, L. L. (1991). Science teaching beliefs and their influence on curriculum implementation: two case studies. Journal of Research in Science Reaching, 38(3), 235-250.

Da silva, C., Mellado, V., Ruiz, C. y Porlán, R. (2007). Evolution of the concep- tions of a secondary education biology teacher: longitudinal analysis using cognitive maps. Science Education, 91(3), 461-491.

Day, C. (1999). Developing teachers, the challenges of lifelong learning, Londres: Falmer Press.

De Longhi, A. L. (2000). El discurso del profesor y del alumno: análisis didáctico en clases de ciencia. Enseñanza de las Ciencias, 18(2), 201-216.

Dillon, D. R., O’Brien, D. G., Moje, E. B. y Stewart, R. A. (1994). Literacy learning in secondary school science classrooms: a cross-case analysis of three qualitative studies. Journal of Research in Science Teaching, 31(4), 345-362.

Duschl, R. A. y Wright, E. (1989). A case study of high school teachers' decision making models for planning and teaching science. Journal of Research in Science Teaching, 26(6), 467-501.

Freire, A. M. y Chorão, M. F. (1992). Elements for a typology of teachers' conceptions of physics teaching. Teaching and Teacher Education, 8(56), 497-507.

Freitas, M. I., Jiménez, R. y Mellado, V. (2004). Solving physics problems: the conceptions and practice of an experienced teacher and an inexperienced teacher. Research in Science Education, 34(1), 113-133.

Friedrichsen, P. M. y Dana, T. M. (2005). Substantive-level theory of highly regarded secondary biology teachers' science teaching orientations. Journal of Research in Science Teaching, 42(2), 218-244

Furió, C. y Carnicer, J. (2002). El desarrollo profesional del profesor de ciencias mediante tutorías de grupos cooperativos. Estudio de ocho casos. Enseñanza de las Ciencias, 20(1), 47-73. 
Gallagher, J. J. (1991). Prospective and practicing secondary school science teachers' knowledge and beliefs about the philosophy of science. Science Education, 75(1), 121-133.

Gil, D. (1993). Contribución de la historia y de la filosofía de las ciencias al desarrollo de un modelo de enseñanza-aprendizaje. Enseñanza de las Ciencias, 1(2), 197-212.

Gil, D., Furió, C. y Gavidia, V. (1998). El profesorado y la reforma educativa en España. Investigación en la Escuela, 36, 49-64.

Gunstone, R. F. y Northfield, J. R. (1994). Metacognition and learning to teach. International Journal of Science Education, 16(5), 523-537.

Hewson, P. W. (1993). Constructivism and reflective practice in science teacher education. En L. Montero y J. M. Vez (eds.), Las didácticas específicas en la formación del profesorado (pp. 259275). Tórculo, Santiago.

Hewson, P. W. y Hewson, M. G. (1989). Analysis and use of a task for identifying conceptions of teaching science. Journal of Educational for Teaching, 15(3), 191-209.

Huibregtse, I., Korthagen, F. y Wubbels, T. (1994). Physics teachers' conceptions of learning, teaching and professional development. International Journal of Science Education, 16(5), 539-561.

Jackson, P. G. (1991). La vida en las aulas. Madrid: Morata.

Jeanpierre, B., Oberhauser, K. y Freeman, C. (2005). Characteristics of professional development that effect change in secondary science teachers' classroom practices. Journal of Research in Science Teaching, 42(6), 668-690.

Jiménez, R. y Wamba, A. M. (2003). ¿Es posible el cambio de modelos didác- ticos? Obstáculos en profesores de ciencias naturales de educación secundaria. Investigación en la Escuela, 17(1), 113-131.

Jiménez-Aleixandre, M. P. y Reigosa, C. (2006). Contextualizing practices across epistemic levels in the chemistry laboratory. Science Education, 90(4), 707-733.

Kang, N. y Wallace, C. (2005). Secondary science teachers' use of laboratory activities: linking epistemological beliefs, goals, and practices. Science Education, 89(1), 140-165.

Kimble, L. L., Yager, R. E. y Yager, S. O. (2006). Success of a professionaldevelopment model in assisting teachers to change their teaching to match the more emphasis conditions urged in the National Science Standards. Journal of Science Teacher Education, 17(3), 309-322.

Lederman, N. G. (1999). Teachers' understanding of the nature of science and classroom practice: factors that facilitate or impede the relationship. Journal of Research in Science Teaching, 6(8), 916-929.

Lederman, N. G. y Zeidler, D. L. (1987). Science teachers' conceptions of the nature of science: do they really influence teaching behavior? Science Education, 71(5), 721-734.

Lee, O. y Porter, A. C. (1993). A teacher's bounded rationality in middle school science. Teaching and Teacher Education, 9(4), 397-409.

López Ruiz, J. I. (1994). El pensamiento del profesor sobre el conocimiento de los alumnos. Investigación en la Escuela, 22, 58-56.

López Ruiz, J. I. (2000). Del enfoque transmisivo al planteamiento de problemas. El caso de Luis. Qurriculum, 14, 189-207. 
Lorsbach, A. W., Tobin, K., Briscoe, C. y Lamaster, S. U. (1992). An interpretation of assessment methods in middle school science. International Journal of Science Education, 14(3), 305-317.

Lucas, S. y Vasconcelos, C. (2005). Perspectivas no âmbito das práticas letivas: Um estudo com professores do $7^{\circ}$ ano de escolaridade. Revista Electrónica de Enseñanza de las ciencias, 4(3). Artículo 4. Extraído desde: http:// www.saum.uvigo.es/reec

Luna, M. y García, J. E. (2003). La transición hacia el conocimiento profesional deseable. Investigación en la Escuela, 49, 23-38.

Lyons, L. L., Freitag, P. K. y Hewson, P. W. (1997). Dichotomy in thinking, dilemma in actions: researcher and teacher perspectives on a chemistry teaching practice. Journal of Research in Science Teaching, 34(3), 239-254.

Martínez-Losada, C. y García-Barros, S. (2005). Do Spanish secondary school teachers really value different sorts of procedural skills? International Journal of Science Education. 27(7), 827-854.

Marx, R. W., Freeman, J., Krajcik, J. y Blumenfed, P. (1998). Professional development of science education. En B. J. Fraser y K. Tobin (eds.), International Handbook of Science Education. (pp. 667-680). Dordrecht: Kluwer A.P.

Mcrobbie, C. y Tobin, K. (1995). Restraints to reform: the congruence of teacher and students actions in a chemistry classroom. Journal of Research in Science Teaching, 32(4), 373-385.

Mellado, V. (1996). Concepciones y prácticas de aula de profesores de ciencias en formación inicial de primaria y secundaria. Enseñanza de las Ciencias, 14(3), 289-302.
Mellado, V. (2003). Cambio didáctico del profesorado de ciencias experimentales y filosofía de la ciencia. Enseñanza de las Ciencias, 21 (3), 343-358.

Mellado, V., Peme-Aranega, C., Redondo, C. y Bermejo, M. L. (2003). Los mapas cognitivos en el análisis gráfico de las concepciones del profesorado de ciencias experimentales. Campo Abierto, 22, 37-58.

Mellado, V., Ruiz, C., Bermejo, M. L. y Jiménez, R. (2006). Contributions from the philosophy of science to the education of science teachers. Science \& Education, 15(5), 419-445.

Mitchener, C. P. y Anderson, R. D. (1989). Teachers' perspective: developing an implementing an sts curriculum. Journal of Research in Science Teaching, 26(4), 351-369.

Not, L. (1983). Las Pedagogías del conocimiento. México: Fondo de Cultura Económica.

Pacca, J. L., Pacca, A. y Villani, A. (1996). Un curso de actualización y cambios conceptuales en profesores de física. Enseñanza de las Ciencias, 14(1), 25-33.

Pavón, F. (1996). Conocimiento profesional de los profesores de física y química de bachillerato principiantes y con experiencia, en la provincia de Cádiz. Tesis Doctoral Inédita. Universidad de Sevilla.

Peers, C. E., Diezmann, C. M. y Watters, J. J. (2003). Supports and concerns for teacher professional growth during the implementation of a science curriculum innovation. Research in Science Education, 33(1), 89-110.

Peme-Aranega, C. (2001). Utilización del "Inventario de creencias didácticas y epistemológicas (Icde)" en la diferenciación de poblaciones de docentes 
de ciencias del nivel medio (en formación) de la ciudad de Córdoba. Comparación con un grupo de docentes (en ejercicio) de ese Nivel. Enseñanza de la Física, 14(1), 5-26.

Peme-Aranega, C. (2003). Hacia el conocimiento de las creencias epistemológicas y didácticas, explícitas e implícitas y su relación: un estudio de caso de una docente de química de secundaria. Trabajo presentado para acceder al Diploma de Estudios Avanzados inédito. Universidad de Extremadura.

Peme-Aranega, C. (2006 a). El desarrollo profesional del profesorado de ciencias experimentales por medio de un proceso de autorreflexión orientado: estudio longitudinal de casos. Tesis doctoral inédita. Universidad de Extremadura.

Peme-Aranega, C. (2006 b). El desarrollo profesional del profesorado de ciencias experimentales por medio de un proceso de autorreflexión orientado: Estudio longitudinal de casos. Revista de Educación en Biología. 9(2), $56-60$

Peme-Aranega, C. y Baquero, M. E. (2001a). El empleo de Inventarios para la descripción de creencias epistemológicas explícitas de una docente de ciencias en un estudio etnográfico. Comparación con las creencias implícitas. Revista de Educación en Biología, 4(1), $15-24$.

Peme-Aranega, C. y Baquero, M. E. (2001b). La descripción de creencias didácticas explícitas de una docente de ciencias empleando Inventarios como parte de un estudio etnográfico. Comparación con las creencias implícitas. Revista de Educación en Biología, 4(2), 9-17.

Peme-Aranega, C., De Longhi, A. L. y Moreno, A. (2005 a). Estudio longitudinal de las creencias de una docente de Física. El proceso de reflexión orientado acerca de ellas, como mejoramiento de su práctica. CD-Rom Enseñanza de las Ciencias, Número Extra, Año 2005, VII Congreso Internacional sobre Investigación en La Didáctica de las Ciencias, Educación Científica para la Ciudadanía, Trabajo 226.

Peme-Aranega, C., De Longhi, A., Baquero, M., Mellado, V. y Ruiz, C. (2005 b). Creencias explícitas e implícitas, sobre la ciencia y su enseñanza y aprendizaje, de una profesora de química de secundaria. CD-Rom Enseñanza de las Ciencias, Número Extra, Año 2005, VII Congreso Internacional sobre Investigación en La Didáctica de las Ciencias, Educación Científica para la Ciudadanía, Trabajo 95.

Peme-Aranega, C., De Longhi, A. L., Baquero, M. E., Mellado, V. y Ruiz, C. 2007. Creencias explícitas e implícitas, sobre la ciencia y su enseñanza y aprendizaje, de una profesora de química de secundaria. Perfiles Educativos. 0185-2698, XXVIII (114), 131-151.

Peme-Aranega, C., Gerbaudo, S., Ferreyra, A. y Echevarrieta, E. (1999). El proceso de elaboración de un inventario de creencias didácticas y epistemológicas (Icde). Interdisciplinaria, 15 (2-3), 1-37.

Porlán, R. (1989). Teoría del conocimiento, teoría de la enseñanza y desarrollo profesional. Las concepciones epistemológicas de los profesores. Tesis Doctoral inédita. Universidad de Sevilla.

Porlán, R. y Rivero, A. (1998). El conocimiento de los profesores. Sevilla: Diada.

Porlán, R., Rivero, A. y Martín, R. (1997). Conocimiento profesional y epistemología de los profesores-I: teoría, 
métodos e instrumentos. Enseñanza de las Ciencias, 15(2), 155-171.

Pozo, J. I. y Gómez, M. A. (1998). Aprender y enseñar ciencia. Madrid: Morata.

Powell, J. C. y Anderson, R. D. (2002). Changing teachers' practice: curriculum materials and science education reform in the usA. Studies in Science Education, 37(1), 107-136.

De Pro, A. (1999). Planificación de unidades didácticas por los profesores: análisis de tipos de actividades de enseñanza. Enseñanza de las Ciencias, 17(3), 411-429.

De Pro, A. (2006). Perfil de la "Reforma Logse" y perfil de uso: los fundamentos de los proyectos curriculares de Física y Química en centros de secundaria. Enseñanza de las Ciencias, 24(3), 337-356.

Reis, P. y Galvao, C. (2004). The impact of socio-scientific controversias in Portuguese natural science teachers' conceptions and practices. Research in Science Education, 34(2), 153-171.

Reyes, F. y Garritz, A. (2006). Conocimiento pedagógico del contenido de reacción química en profesores universitarios mexicanos. Revista Mexicana de Investigación Educativa, 31(XI), 1175-1205.

Reyes, L., Salcedo, L. E. y Perafán, G. (2001). Acciones y creencias IV: análisis e interpretación de creencias de docentes de biología y ciencias naturales. Bogotá: Universidad Pedagógica Nacional.

Rodríguez, P., López, J. y Mota, A. (2006). ¿Cómo se articulan las concepciones epistemológicas y de aprendizaje con la práctica docente en el aula? Revista Mexicana de Investigación Educativa, 31(11), 1307-1335.
Roth, W. M. (1998). Science teaching as knowledgability: a case study of knowing and learning during coteaching. Science Education, 82(3), 357-377.

Sánchez, G. y Valcárcel, M. V. (2000). ¿Qué tienen en cuenta los profesores cuando seleccionan el contenido de enseñanza? Cambios y dificultades tras un programa de formación. Enseñanza de las Ciencias, 18(3), 423-437.

Sanmartí, N. (2001). Enseñar a enseñar ciencias en secundaria: un reto muy complejo. Revista Interuniversitaria de Formación del Profesorado, 40, 31-48.

Sassi, E., Monroy, G. y Testa, I. (2005). Teacher training about real-time approaches: Research-based guidelines and training materials. Science Education, 89(1), 28-37.

Shulman, L. S. (1986). Those who understand: knowledge growth in teaching. Educational Researcher, 15(2), 4-14.

Shwartz, Y., Ben-Zvi, R. y Hofstein, A. (2005). The importance of involving high-school chemistry teachers in the process of defining the operational meaning of "chemical literacy". International Journal of Science Education, 27(3), 323-344.

Tal, R. T., Dori, Y. J., Keiny, S. y Zoller, U. (2001). Assessing conceptual change of teachers involved in sTEs education and curriculum development. The STEMS project approach. International Journal of Science Education, 23(3), 247-262.

Tobin, K. (1998). Issues and trends in the teaching of science. En B. J. Fraser y K.

Tobin (eds.), International handbook of science education (pp. 129-151). Dordrecht: Kluwer A. P. 
Tobin, K. y Espinet, M. (1989). Impediments to change: applications of coaching in high school science teaching. Journal of Research in Science Teaching, 26(2), 105-120.

Tobin, K. y McRobbie, C. (1996). Cultural myths as constraints to the enacted science currículo. Science Education, 80(2), 223-241.

Tsai, C.C. (2002). Nested epistemologies: science teachers' beliefs of teaching, learning and science. International Journal of Science Education, 24(8), 771-783

Valcárcel, M. V. y Sánchez, G. (2000). La formación del profesorado en ejercicio. En F. J. Perales y P. Cañal (eds.), Didáctica de las ciencias experimentales (pp. 557-581). Alcoy: Marfil.

Vázquez, B., Jiménez, R. y Mellado, V. (2007). La reflexión en profesoras de ciencias experimentales de enseñanza secundaria. Estudio de casos. Enseñanza de las Ciencias, 25(1), 73-90.
Vázquez, B., Jiménez, R., Mellado, V., Martos, M. y Taboada, C. (2006). Evolución de la reflexión y práctica de aula en la resolución de problemas. El caso de dos profesoras de ciencias de secundaria. Campo Abierto, 25(1), 133-152.

Verjovsky, J. y Waldegg, G. (2005). Analyzing beliefs and practices of a Mexican high school biology teacher. Journal of Research in Science Teaching, 42(4), 465-491.

Wallace, C. S. y Kan, N. H. (2004). An investigation of experienced secondary science teachers' beliefs about inquiry: an examination of competing belief sets. Journal of Research in Science Teaching, 41(1), 936-960.

White, R. T. y Arzi, H. J. (2005). Longitudinal studies: designs, validity, practicality, and value. Research in Science Education, 35(1), 137-149. 